\title{
Processos quase estáticos, reversibilidade e os limites da Termodinâmica
}

\author{
Quasi-static processes, reversibility and the limits of thermodynamics \\ Sinara Santos Dourado ${ }^{1}$, Marcelo Amorim Marchiori*1] \\ ${ }^{1}$ Universidade Federal de Mato Grosso, Instituto de Física, Cuiabá, MT, Brasil
}

Recebido em 05 de Março, 2018. Revisado em 28 de Agosto, 2018. Aceito em 03 de Setembro, 2018.

\begin{abstract}
Este trabalho apresenta, por meio do formalismo clássico dos cursos de termodinâmica, um estudo da relação entre a natureza quase estática de um processo termodinâmico e sua reversibilidade. Além disso, a partir dos resultados obtidos ao supor a reversibilidade para um processo termodinâmico composto por um subprocesso fundamentalmente irreversível, exploramos os limites de aplicação das leis da termodinâmica e as nuances contidas nas suas formulações gerais. Consideramos que este artigo possui dois caráteres bem definidos: o didático e o fundamental. O caráter didático fica evidente quando utilizamos as técnicas de cálculo para a extração das quantidades termodinâmicas nos processos infinitesimais e ao colocar o leitor em contato com as técnicas de demonstração por contradição (se uma hipótese leva a uma contradição, ela não pode ser tomada). Ambas as técnicas podem ser vistas como essenciais na abordagem de um número imenso de problemas em Física. Do ponto de vista fundamental, conseguimos evidenciar a íntima relação entre a Primeira e a Segunda leis da Termodinâmica quando lidamos com a determinação das condições de reversibilidade e existência dos processos termodinâmicos. Palavras-chave: Termodinâmica, Reversibilidade, Quase estático, Irreversibilidade.
\end{abstract}

This work presents, by means of the classical formalism of thermodynamics courses, a study of the relation between the quasi-static nature of a thermodynamic process and its reversibility. In addition, from the results obtained by assuming the reversibility of a thermodynamic process composed by a fundamentally irreversible sub-process, we explore the limits of application of the Laws of Thermodynamics and the nuances contained in its general formulations. We consider that this paper has two well defined purposes: didactic and fundamental. The didactic point of view is evident when we use the calculus techniques for the extraction of the thermodynamic quantities in the infinitesimal processes and by putting the reader in contact with the techniques of proof by contradiction (if a hypothesis leads to a contradiction, then it can not be assumed). Both techniques can be seen as essential in the approach of a huge number of problems in physics. From the fundamental point of view, we have been able to evidence the intimate relationship between the First and Second Laws of Thermodynamics when we deal with the determination of the conditions of reversibility and existence of thermodynamic processes. Keywords: Thermodynamics, Reversibility, Quasi-static, Irreversibility.

\section{Introdução}

A irreversibilidade é uma manifestação de qualquer processo físico real [1],2]. Por irreversibilidade entendemos a impossibilidade de execução de um processo físico no sentido inverso ao sentido temporal original. Como exemplo, podemos citar a impossibilidade de transferência de calor espontaneamente de um corpo frio para um corpo quente. A natureza é repleta de processos irreversíveis e eles são muito mais comuns do que os processos reversíveis. Estes últimos, por sua vez, são raros e dependem de condições muito singulares para ocorrerem. No entanto, mesmo diante da natureza irreversível da dinâmica de sistemas reais, toda a teoria da termodinâmica clássica ensinada nos cursos de graduação está baseada na hipótese de equilíbrio e reversibilidade destes processos.

*Endereço de correspondência: marchiori@fisica.ufmt.br
Portanto, grandezas como energia interna $(U)$ e entropia $(S)$ somente podem ser calculadas a partir de processos reversíveis de equilíbrio. Nos casos irreversíveis, como a expansão livre, o cálculo das grandezas termodinâmicas entre dois estados é feito a partir de processos reversíveis que conectem estes mesmos estados.

Na maioria dos casos, devemos impor a condição de reversibilidade por meio de restrições ou idealizações aos processos físicos reais e a validade destes procedimentos pode ser atestada a partir da concordância entre os resultados teóricos e experimentais [1]2. É neste ponto que reside o triunfo da Termodinâmica: as suas principais leis têm origem em resultados experimentais amplamente verificados. Por esta razão, é uma teoria extremamente bem sucedida e constitui um dos principais pilares da Física. Porém, devemos sempre ter em mente que algumas das restrições ou idealizações associadas às condições de re- 
versibilidade e equilíbrio podem não ser válidas em algum processo específico e, portanto, assumi-las indiscriminadamente poderia gerar algum tipo de contradição nos resultados. Em parte é disto que este artigo se ocupa: verificar a validade da hipótese de que a reversibilidade está associada à natureza quase estática dos processos termodinâmicos, uma afirmação muito comum nos livros [1 5$]$. O outro foco deste trabalho é o limite de aplicação das formas gerais das leis da termodinâmica. Em outras palavras, buscamos mostrar, primeiramente, se a natureza quase estática é uma condição suficiente para a reversibilidade e, logo após, verificar, através da construção de um processo cíclico específico, que as formulações gerais da Primeira e Segunda leis devem ser utilizadas com muito cuidado. É por esta razão que faremos uma breve revisão dos conteúdos das leis da Termodinâmica.

A Termodinâmica possui um conjunto de quatro leis empíricas, ou seja, impostas pela natureza e descritas a partir de experimentos. A Lei Zero define o protocolo de utilização de termômetros e, portanto, estabelece as regras para medir a temperatura e estabelecer suas escalas [2, 4]. A $3^{\text {a }}$ Lei trata do que acontece a sistemas que estão suficientemente próximos do zero absoluto [4] e está fora do escopo deste trabalho. Desta forma, focaremos nossa atenção na Primeira e Segunda leis. Para fins didáticos, a seguir, faremos uma breve exposição de como as formas gerais destas leis decorrem de resultados experimentais.

A Primeira Lei da Termodinâmica, em sua forma experimental, pode ser enunciada como segue:

\section{Enunciado da $1^{\mathrm{a}}$ Lei da Termodinâmica:}

\section{"O trabalho realizado de forma adiabática entre dois estados bem definidos independe do processo 4 .".}

Um processo adiabático é realizado de forma isolada, ou seja, sem que haja qualquer troca de energia com o meio externo. Assim, um trabalho adiabático só pode ser realizado à custa da energia interna $(U)$. Desta forma, podemos escrever

$$
\Delta U=-W_{A d}
$$

com $W_{A d}$ sendo o trabalho adiabático. O sinal negativo indica que um trabalho positivo, realizado pelo sistema, implica um decréscimo da energia interna. Como consequência, se permitirmos a realização de trabalho $(W)$ conjuntamente com fluxo de energia externa, é possível definir o calor $(Q)$ como a diferença entre o trabalho realizado (ou sofrido) e a variação da energia interna, ou seja,

$$
W-W_{A d}=Q .
$$

Utilizando a igualdade (1) obtemos

$$
\Delta U=Q-W,
$$

a formulação geral da Primeira Lei da termodinâmica. Nos processos cíclicos, aqueles em que os estados inicial e final coincidem, devemos ter

$$
\Delta U=0
$$

sempre. Este resultado decorre imediatamente do enunciado da Primeira Lei. Diz-se, portanto, que a energia interna é uma propriedade termodinâmica independente do caminho.

A $2^{\mathbf{a}}$ Lei da Termodinâmica, por sua vez, em sua forma experimental, pode ser enunciada de duas maneiras:

\section{Enunciado de Kelvin-Planck:}

"É impossível realizar um processo cíclico cujo único efeito seja remover calor de um reservatório térmico e produzir uma quantidade equivalente de trabalho [4]."

\section{Enunciado de Clausius:}
"É impossível realizar um processo cíclico cujo único efeito seja transferir calor de um corpo mais frio para um corpo mais quente [4]."

Em linhas gerais, ambos os enunciados tratam de limites impostos aos processos cíclicos, isto é, processos que retornam ao estado inicial do sistema. De fato, o enunciado de Kelvin-Planck afirma que é impossível em um processo cíclico realizar trabalho utilizando toda a energia extraída de um reservatório térmico, deve sempre haver uma parcela descartada para um reservatório mais frio. O enunciado de Clausius, por outro lado, afirma que existe uma "direção" obrigatória para o fluxo espontâneo de calor (sem realização de trabalho): sempre do corpo mais quente para o corpo mais frio. Apesar de parecerem independentes, os dois enunciados são equivalentes [2, 4 .

Durante a revolução industrial europeia, a busca contínua pelos limites de eficiência máxima de uma máquina conduziu o engenheiro Sadi Carnot à idealização de um processo cíclico conhecido como Ciclo de Carnot 2, 4 e o consequente enunciado do resultado:

\section{Teorema de Carnot}

(i) "Nenhuma máquina térmica que opere entre uma dada fonte quente e uma dada fonte fria pode ter rendimento superior ao de uma máquina de Carnot [4]."

(ii) "Todas as máquinas de Carnot que operem entre essas duas fontes terão o mesmo rendimento 4]."

A máquina de Carnot é uma máquina idealizada, uma vez que é composta somente por processos reversíveis. O processo cíclico associado à máquina de Carnot é o ciclo de Carnot e pode ser caracterizado pelas seguintes fases:

(1) extração isotérmica de calor de um reservatório quente $\left(T_{1}\right)$; 
(2) uma fase de realização de trabalho sem fluxo de calor (trabalho adiabático);

(3) rejeição isotérmica de calor para um reservatório frio $\left(T_{2}\right)$;

(4) uma fase em que o sistema sofre trabalho adiabático e retorna ao volume inicial.

O Teorema de Carnot é equivalente aos enunciados de Kelvin-Planck e Clausius, pois a violação de um implica a violação do outro 4]. Logo, o limite imposto pela eficiência do ciclo de Carnot também pode ser visto como um enunciado da Segunda Lei da Termodinâmica. Utilizando o resultado de Carnot pode-se mostrar que, para processos cíclicos reversíveis arbitrários, existe uma grandeza termodinâmica que, assim como a energia interna, dados os estados inicial e final de equilíbrio, é independente do caminho. Esta grandeza é denominada entropia e sua variação é definida por

$$
\Delta S=\int_{i}^{f} \frac{d Q}{T} .
$$

A partir da desigualdade de Clausius [2, 4],

$$
\oint \frac{d Q}{T} \leqslant 0
$$

verifica-se que, para sistemas fechados, vale

$$
\Delta S \geqslant 0
$$

A equação 6) resume o princípio de aumento da entropia. A igualdade é válida para processos reversíveis, enquanto a desigualdade está associada a processos irreversíveis. A expressão (6) pode ser vista como a formulação geral da Segunda Lei da Termodinâmica 2] .

A expressão (6) estabelece a condição para que haja reversibilidade $(\Delta S=0)$. Por sua vez, a reversibilidade termodinâmica tem estreita ligação com a velocidade de execução do processo. Os livros textos dos cursos de graduação, em geral, explicitam a necessidade de variação de estado suficientemente lenta, de forma que o tempo de realização do processo seja muito maior do que os tempos característicos de relaxação 1,2]. Por outro lado, a realização de forma quase estática implica uma baixa taxa de trabalho por unidade de tempo, ou seja, os processos realizados desta maneira têm baixa potência [6 10]. Existe um grande número de trabalhos que tratam da correlação entre a eficiência de uma máquina e a velocidade do processo $7,9,11$. Inclusive, a velocidade de execução é um dos elementos centrais nos processos de computação reversível 12 14 .

Embora seja extremamente relevante, não nos focaremos no estudo da potência de processos quase estáticos e sim na questão fundamental de verificar se no limite quase estático é possível garantir a execução reversível de todo processo termodinâmico. Com este fim, inicialmente, reproduzimos os resultados associados ao limite de eficiência de um motor simples operando entre dois reservatórios com capacidade térmica finita sem qualquer restrição com respeito ao tempo de execução. Este é um problema clássico dos cursos básicos 11. Posteriormente, no Caso 1, verificamos que este limite é alcançado ao impormos a discretização do processo em etapas operadas por máquinas de Carnot que produzem variações infinitesimais de temperatura em cada um dos reservatórios. Desta forma garantimos a execução quase estática do processo. No Caso 2, estudamos as consequências da realização do mesmo processo sob a ação de expansões livres adiabáticas (irreversíveis) de forma quase estática. Por fim, a partir do Caso 3 e ao assumirmos, por hipótese, que o limite quase estático elimina a irreversibilidade do Caso 2, mostramos que ocorre uma violação da Primeira Lei da Termodinâmica. Além disso, verificamos que as formas gerais das leis da termodinâmica precisam ser analisadas conjuntamente e com muito mais cuidado do que usualmente é feito nos livros textos. Mostramos que o limite de reversibilidade imposto somente pela Segunda Lei não é capaz de impedir, no processo estudado, que a eficiência do motor seja maior em comparação ao caso reversível (Caso 1). E ainda, evidenciamos que nem a Primeira ou a Segunda Lei, em suas formas gerais, impedem a existência de um processo irreversível que apresente uma eficiência igual à do Caso 1 .

\section{Modelo}

Com o intuito de demonstrar que a natureza quase estática de um processo não é uma condição suficiente para a reversibilidade, supomos que um motor genérico opere entre dois reservatórios térmicos finitos, 1 e 2, compostos por um mesmo gás ideal com capacidades térmicas $C$, cujas temperaturas iniciais são, respectivamente, $T_{1}$ e $T_{2}$, sendo $T_{1}>T_{2}$. Este sistema está ilustrado na Figura 1 .

Este é um problema clássico de livros textos [1] e é possível, utilizando a Primeira e Segunda leis da Termodinâmica, estabelecer um limite superior para o trabalho realizado pelo motor (para uma dedução completa ver o Apêndice A). De fato, mostra-se que o trabalho realizado está limitado por

$$
W \leqslant C\left(T_{1}+T_{2}-2 \sqrt{T_{1} T_{2}}\right)
$$

onde $C=N_{0} C_{V}$, sendo $N_{0}$ o número de mols que compõem o gás contido em cada reservatório e $C_{V}$ seu calor

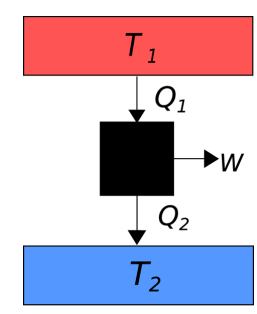

Figura 1: Motor térmico operando entre dois reservatórios térmicos finitos com capacidade térmica $C$. Os reservatórios 1 e 2 foram representados nas cores vermelho e azul, respectivamente. 
específico molar a volume constante. Portanto, podemos concluir que

\section{Para que a Segunda Lei da Termodinâmica seja respeitada, o trabalho realizado por qual- quer máquina que opere entre os mesmos reservatórios, não excederá ao limite superior imposto pela expressão (7). O limite superior somente poderá ser alcançado nos processos reversíveis de eficiência máxima.}

Para obter o caso reversível de máxima eficiência, a igualdade em (7), dividiremos o processo em sucessões infinitesimais com $N$ etapas e em cada uma delas utilizaremos um motor de Carnot como sistema que realiza trabalho, como ilustra a Figura 2 Utilizando este modelo geral estudaremos três tipos de processos:

- Caso 1: Operação dos motores de Carnot, fazendo com que a variação de temperatura do reservatório $1\left(\Delta T_{1}\right)$ entre cada n-ésima fase do processo seja sempre a mesma, o que implica a extração de uma quantidade fixa de calor, $Q=N_{0} C_{V} \Delta T_{1}$, em cada fase. $\mathrm{O}$ volume dos reservatórios permanece constante.

- Caso 2: Operação dos motores de Carnot extraindo do reservatório 1 uma quantidade fixa de calor $Q=N_{0} C_{V} \Delta T_{1}$ em cada fase do processo e permitindo ao reservatório 1 sofrer uma expansão livre adiabática entre cada um dos ciclos do motor.

- Caso 3: Operação de refrigeradores de Carnot partindo do estado final do Caso 2 e recuperando o estado inicial do sistema por meio de contrações infinitesimais isotérmicas do reservatório 1.

\section{Resultados e Discussões}

Apresentaremos, a seguir, o tratamento de cada um dos três casos descritos na seção 2

\subsection{Caso 1: Extração de energia constante e o limite quase estático dos ciclos de Carnot}

O principal objetivo da análise deste caso é verificar que, no limite quase estático, é possível atingir o limite im- posto pela Segunda Lei da Termodinâmica para eficiência de um motor térmico. Isto corresponde à máxima realização de trabalho pelo sistema, ou seja, quando verifica-se a igualdade na equação (7).

O sistema parte de seu estado inicial e alcança o estado final por meio de $N$ etapas. Os reservatórios partem de suas temperaturas iniciais $T_{10}=T_{1}$ e $T_{20}=T_{2}$ e o processo é conduzido até que ambos os reservatórios possuam a mesma temperatura $T_{F}^{(1)}$. O trabalho total realizado pelo motor é a soma dos trabalhos realizados em cada uma das etapas, ou seja,

$$
W^{(1)}=\sum_{n=0}^{N} W_{n}^{(1)} .
$$

A eficiência de um motor arbitrário é definida pela expressão

$$
\eta=\frac{|W|}{\left|Q_{1}\right|}=1-\frac{\left|Q_{2}\right|}{\left|Q_{1}\right|}
$$

onde $Q_{1}$ e $Q_{2}$ são as quantidades de calor absorvido da fonte 1 e liberado para a fonte 2 , respectivamente, e $W=$ $\left|Q_{1}\right|-\left|Q_{2}\right|$. Como estamos interessados em maximizar a eficiência do processo entre os reservatórios dados, utilizaremos um motor de Carnot, cuja a eficiência na n-ésima fase é dada por

$$
\eta_{n}=1-\frac{T_{2 n}}{T_{1 n}},
$$

onde $T_{1 n}$ e $T_{2 n}$ correspondem às temperaturas das fontes 1 e 2 , respectivamente, na n-ésima fase do processo [4]. Como em cada etapa é extraída uma quantidade fixa de calor do reservatório $1, Q_{1_{n}}=N_{0} C_{V} \Delta T_{1}$, produzimos sempre a mesma variação de temperatura $\left(\Delta T_{1}\right)$ neste reservatório. Utilizando as equações (9) e $(10)$ e lembrando que $\left|Q_{1_{n}}\right|=-Q_{1_{n}}$ e $\left|Q_{2_{n}}\right|=Q_{2_{n}}$, pois $\Delta T_{1}<0$ e $\Delta T_{2}>0$, podemos escrever que o trabalho realizado pelo motor de Carnot na n-ésima etapa do processo será

$$
W_{n}^{(1)}=-\left(1-\frac{T_{2 n}}{T_{1 n}}\right) N_{0} C_{V} \Delta T_{1} .
$$

Por outro lado, ao compararmos a segunda igualdade da equação (9) e a 10 , usando $Q_{2_{n}}=N_{0} C_{V} \Delta T_{2 n}$,

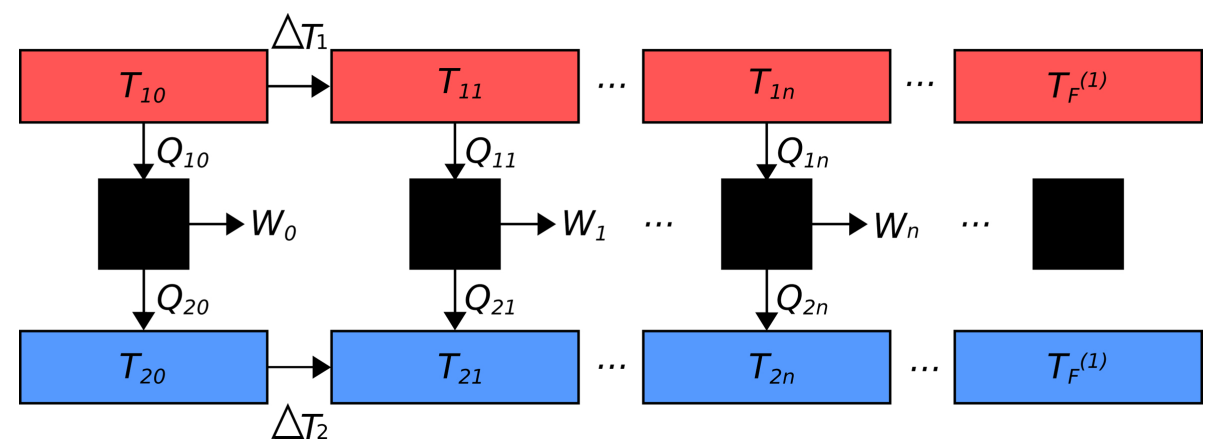

Figura 2: Sucessão de processos divididos em $N$ etapas discretas e infinitesimais, realizadas por máquinas de Carnot, que levam os reservatórios de $T_{10}=T_{1}$ e $T_{20}=T_{2}$ a $T_{F}^{(1)}$. Os reservatórios 1 e 2 foram representados nas cores vermelho e azul, respectivamente. 
encontramos a relação entre as variações de temperatura nos reservatórios 1 e 2 ,

$$
\Delta T_{2 n}=-\frac{T_{2 n}}{T_{1 n}} \Delta T_{1}
$$

Resta-nos, neste ponto, determinar as condições para que o processo seja realizado de forma quase estática, ou seja, de maneira que o tempo de realização de cada etapa seja suficientemente longo quando comparado às escalas do tempo de relaxação do sistema termodinâmico. No entanto, como o tempo não é uma grandeza que aparece explicitamente no problema, a natureza quase estática é definida a partir de variações infinitesimais dos parâmetros termodinâmicos 1,2. Neste caso, tomaremos como infinitesimal a quantidade de calor extraída do reservatório 1 , o que significa dizer que a variação de temperatura será infinitesimal. Matematicamente, o trabalho total realizado pelos motores de Carnot no processo quase estático completo pode ser escrito como

$$
W^{(1)}=\lim _{\substack{N \rightarrow \infty \\ \Delta T \rightarrow 0}} \sum_{n=0}^{N} W_{n}^{(1)} .
$$

No limite infinitesimal $(\Delta T \rightarrow 0)$ podemos reescrever as equações (11) e 12 como

$$
d W=-\left(1-\frac{T_{2}^{\prime}}{T_{1}^{\prime}}\right) N_{0} C_{V} d T_{1}^{\prime}
$$

e

$$
d T_{2}^{\prime}=-\frac{T_{2}^{\prime}}{T_{1}^{\prime}} d T_{1}^{\prime}
$$

Logo, de 14, verificamos que

$$
W^{(1)}=\int_{T_{1}}^{T_{F}^{(1)}}-N_{0} C_{V} d T_{1}^{\prime}-\int_{T_{2}}^{T_{F}^{(1)}} N_{0} C_{V} d T_{2}^{\prime},
$$

o que implica

$$
W^{(1)}=N_{0} C_{V}\left(T_{1}+T_{2}-2 T_{F}^{(1)}\right) .
$$

O valor de $T_{F}^{(1)}$ é obtido a partir da integração da equação 15 e resulta em

$$
T_{F}^{(1)}=\sqrt{T_{1} T_{2}}
$$

o que significa

$$
W^{(1)}=W_{\text {Caso } 1}^{\text {Máx }}=N_{0} C_{V}\left(T_{1}+T_{2}-2 \sqrt{T_{1} T_{2}}\right) .
$$

onde $W_{\text {Caso } 1}^{\text {Máx }}$ corresponde à igualdade da equação (7), ou seja, à quantidade máxima de trabalho que pode ser obtida para o sistema considerado. Cabe aqui um comentário importante. O limite em que $\Delta T \rightarrow 0$ é o que garante a reversibilidade do processo e que os ciclos infinitesimais sejam conduzidos por motores de Carnot legítimos, pois é somente neste limite que garantimos a extração e liberação isotérmica de calor para os reservatórios 1 e 2, respectivamente. Assim, o resultado (18) resume a constatação de que ao utilizarmos o motor de máxima eficiência possível entre dois reservatórios térmicos, o motor de Carnot, é possível alcançar o limite imposto pela Segunda Lei da Termodinâmica, o que significa alcançar o limite associado ao processo reversível de eficiência máxima. Além disso, como qualquer processo cíclico reversível pode ser decomposto em uma sucessão infinitesimal de ciclos de Carnot, seria natural concluir que:

\section{Tomar o limite infinitesimal (quase estático) de operação de um motor implica tornar o processo reversível.}

No entanto, mostraremos nas próximas seções, que o limite de execução quase estático não é uma condição suficiente para garantir a reversibilidade do processo.

\subsection{Caso 2: Trabalho máximo de uma máquina operando entre reservatórios finitos realizando expansão livre adiabática}

Pela resolução do caso anterior encontramos que, em princípio, a imposição de que o processo seja realizado de maneira quase estática resulta na reversibilidade do mesmo. Para testar a veracidade desta afirmação estudamos uma variante do problema descrito no Caso 1. Em cada etapa do processo do caso anterior, além das transferências de calor já descritas, permitimos que o reservatório 1 sofra uma expansão livre adiabática infinitesimal, de tal forma que o volume original varie de $V_{n}$ para $V_{n+1}=V_{n}+\Delta V_{n}$, como ilustrado na Figura 3 . Como a expansão livre adiabática não muda a energia interna do gás, sua temperatura permanece a mesma e, desta forma, garantimos em cada fase de execução as mesmas temperaturas do caso anterior.

Em geral, encontra-se nos livros didáticos 4, o cálculo da variação de entropia no processo de expansão livre adiabática $\left(\Delta S_{E}\right)$, dada por

$$
\Delta S_{E}=N_{0} R \ln \left(\frac{V_{f}}{V_{i}}\right)>0,
$$

onde $V_{i}$ e $V_{f}$ são, respectivamente, os volumes inicial e final do sistema que realiza a expansão livre adiabática. Desta forma, a variação de entropia da n-ésima etapa do processo será

$$
\Delta S_{E}^{(n)}=N_{0} R \ln \left(\frac{V_{n+1}}{V_{n}}\right) .
$$

A entropia total gerada pelas expansões livres é, portanto, a soma da variação em cada etapa particular

$$
\Delta S_{E}^{(T)}=\sum_{n=0}^{N} \Delta S_{E}^{(n)}=\sum_{n=0}^{N} N_{0} R \ln \left(\frac{V_{n+1}}{V_{n}}\right)
$$




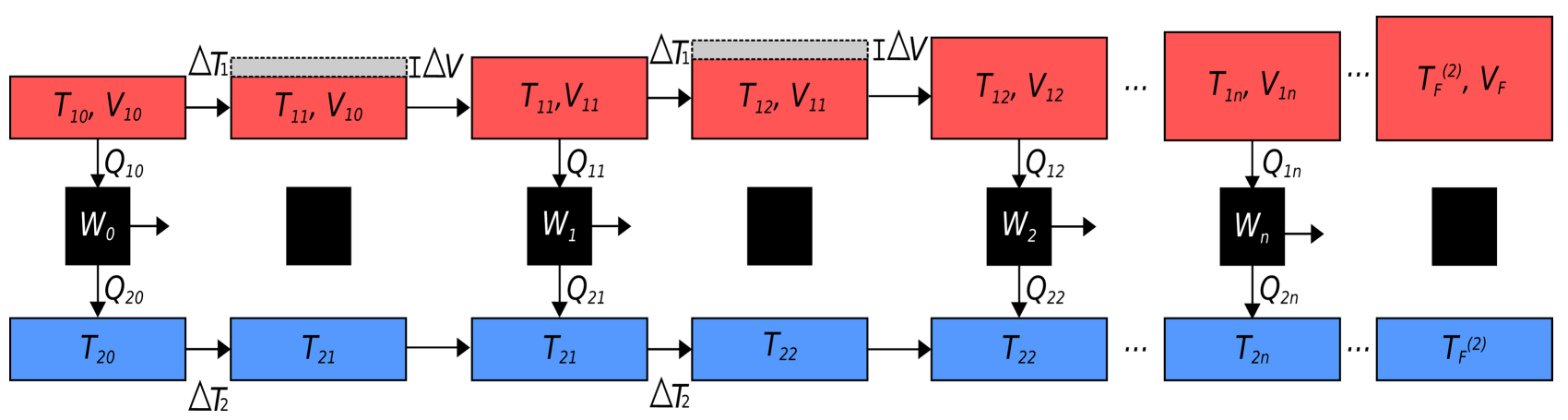

Figura 3: Processo em que o reservatório 1 realiza expansão livre adiabática. Os reservatórios 1 e 2 foram representados nas cores vermelho e azul, respectivamente.

Usando as propriedades da operação logarítmica, obtemos

$$
\Delta S_{E}^{(T)}=N_{0} R \ln \left(\frac{V_{F}}{V_{0}}\right)
$$

ou seja, depende, como esperado, apenas dos estados final e inicial. A expansão livre adiabática de um gás isolado é fundamentalmente irreversível $\left(\Delta S>0\right.$, pois $\left.V_{F}>V_{0}\right)$, ou seja, independe se o processo é realizado de maneira abrupta ou de forma quase estática. Podemos notar isso pelo fato de que a expressão (19), que não apresenta restrições quanto à velocidade do processo, é semelhante à expressão (22), uma vez que só depende dos volumes inicial e final do processo. Entretanto, como neste caso o reservatório que sofre a expansão livre adiabática não está isolado, a expressão (22) não é suficiente para garantir a irreversibilidade, dado que a forma geral da Segunda Lei exige que o sistema seja fechado. Neste caso, como o motor se acopla ao reservatório para extração de energia, é necessário calcular a entropia no processo como um todo, ou seja, devemos somar a entropia decorrente da produção de trabalho, $\Delta S_{W}^{(T)}$, dada pela equação (A10), e a entropia associada à expansão livre, $\Delta S_{E}^{(T)}$, dada pela equação (22). Assim

$$
\Delta S^{(2)}=\Delta S_{W}^{(T)}+\Delta S_{E}^{(T)} .
$$

Pela Segunda Lei da Termodinâmica, para o sistema isolado, devemos ter

$$
\Delta S^{(2)} \geqslant 0,
$$

o que, segundo as equações (A10) e 222 , implica

$$
N_{0} C_{V} \ln \left(\frac{\left(T_{F}^{(2)}\right)^{2}}{T_{1} T_{2}}\right)+N_{0} R \ln \left(\frac{V_{F}}{V_{0}}\right) \geqslant 0 .
$$

Isolando $T_{F}^{(2)}$, obtemos

$$
T_{F}^{(2)} \geqslant\left(T_{1} T_{2}\right)^{\frac{1}{2}}\left(\frac{V_{0}}{V_{F}}\right)^{\frac{R}{2 C_{V}}}
$$

como o novo limite para a temperatura final de equilíbrio dos reservatórios. Substituindo a equação 26 em (16), encontramos que o limite imposto pela segunda lei da termodinâmica para o trabalho realizado pelo sistema será

$$
W^{(2)} \leqslant N_{0} C_{V}\left(T_{1}+T_{2}-2\left(T_{1} T_{2}\right)^{\frac{1}{2}}\left(\frac{V_{0}}{V_{F}}\right)^{\frac{R}{2 C_{V}}}\right) .
$$

Portanto:

$$
\begin{aligned}
& \text { Para que a Segunda Lei da Termodinâmica } \\
& \text { seja respeitada, o trabalho realizado por qual- } \\
& \text { quer máquina que opere entre os mesmos } \\
& \text { reservatórios e sujeita aos mesmos processos } \\
& \text { de expansão livre adiabática, não excederá ao } \\
& \text { limite superior imposto pela expressão } 27 \text {. } \\
& \text { O limite superior somente poderá ser alcan- } \\
& \text { cado nos processos reversíveis de eficiência } \\
& \text { máxima. }
\end{aligned}
$$

O limite superior, ou seja, a igualdade na equação (27), somente pode ser alcançada se houver reversibilidade. Sabemos que a expansão livre adiabática de um gás isolado ocorre de tal forma que $\Delta S>0$ e gostaríamos de avaliar quais as consequências de se assumir que o processo completo, realizado de forma infinitesimal (quase estática), pode ser considerado reversível. Em outras palavras, gostaríamos de avaliar quais as implicações se assumirmos que a natureza quase estática do processo é capaz de tornar a expansão livre reversível. Esta é a essência da demonstração por absurdo: assumimos como verdadeira a hipótese falsa e buscamos as contradições e absurdos decorrentes de assumi-la como verdadeira.

Com isto em mente, vamos considerar a igualdade na equação (27) e comparar as eficiências das máquinas (equação (9)) em cada caso. Começaremos comparando os trabalhos realizados em cada um dos processos infinitesimais, ou seja, quando mantemos o volume do reservatório 1 constante (Caso 1) e quando permitimos a expansão livre do mesmo (Caso 2). Na expansão, como $V_{0}<V_{F}$, a constante dos gases $R$ e o calor específico molar a volume constante $C_{V}$ são números positivos, podemos escrever

$$
\left(\frac{V_{0}}{V_{F}}\right)^{\frac{R}{2 C_{V}}}<1
$$


que resulta em

$$
2 \sqrt{T_{1} T_{2}}\left(\frac{V_{0}}{V_{F}}\right)^{\frac{R}{2 C_{V}}}<2 \sqrt{T_{1} T_{2}}
$$

e, por fim, temos a seguinte desigualdade

$C\left(T_{1}+T_{2}-2 \sqrt{T_{1} T_{2}}\left(\frac{V_{0}}{V_{F}}\right)^{\frac{R}{2 C_{V}}}\right)>C\left(T_{1}+T_{2}-2 \sqrt{T_{1} T_{2}}\right)$,

com $C=N_{0} C_{V}$. O lado esquerdo da equação acima é equivalente à igualdade da inequação (27) e corresponde ao limite do trabalho máximo no caso em que se considera a expansão livre adiabática $\left(W_{\text {Caso } 2}^{\text {Máx }}\right)$. O lado direito de (28) é igual ao limite do trabalho máximo no processo reversível original $\left(W_{\text {Caso } 1}^{\text {Máx }}\right)$, dado pela igualdade na equação 18). Logo, chegamos à conclusão de que

$$
W_{\text {Caso 2 }}^{\text {Máx }}>W_{\text {Caso 1 }}^{\text {Máx }},
$$

ou seja, o limite de trabalho útil quando há a expansão livre adiabática é maior do que o limite do trabalho útil quando o processo é composto somente pela atuação de máquinas de Carnot reversíveis. Este resultado é contraintuitivo, pois seria natural acreditar que haveria uma diminuição do trabalho máximo realizado pelo motor, uma vez que inserimos em cada etapa de operação um processo fundamentalmente irreversível, a expansão livre adiabática infinitesimal. É muito importante ter em mente de que estamos em busca de uma contradição que invalide a hipótese de que o processo com expansão livre quase estática (Caso 2) pode ser considerado um processo reversível. A relação 290 é validada pela Segunda Lei da Termodinâmica, logo a análise isolada da condição $\Delta S \geqslant 0$ não impede que o limite máximo de trabalho útil aumente no Caso 2.

Outra propriedade relevante que deve ser comparada é a eficiência dos processos caso assumamos a reversibilidade de ambos. Para determiná-las é necessário calcular as quantidades de calor $\left(Q_{1}^{(i)}\right)$ extraídas do reservatório 1 em cada caso. Temos, portanto

$$
Q_{1}^{(i)}=N_{0} C_{V}\left(T_{F}^{(i)}-T_{10}\right),
$$

onde i se refere aos Casos 1 e 2.

A partir das inequações (26) e (A12), ao supormos que os Casos 1 e 2 são reversíveis, podemos ver que $T_{F}^{(2)}<T_{F}^{(1)}$. As eficiências de ambos os casos foram calculadas e comparadas no Apêndice B, como resultado obtemos

$$
\eta_{\text {Caso 2 }}^{\text {Máx }}>\eta_{\text {Caso 1 1 }}^{\text {Máx }} \text {. }
$$

Os resultados 29) e (31) são absolutamente inesperados, pois ao introduzirmos um processo essencialmente irreversível, a Segunda Lei da da Termodinâmica, por si só, não garante a diminuição da eficiência do processo. Somado a este surpreendente resultado quando se considera a igualdade $\Delta S^{(2)}=0$, é muito importante ressaltar que a utilização da forma geral $\left(\Delta S^{(2)} \geqslant 0\right)$ não é capaz de invalidar os resultados das equações 29) e (31) mesmo nos casos em que não assumimos a reversibilidade do Caso 2. Isto significa que qualquer trabalho $\left(W^{(2)}\right)$ realizado pelo sistema de tal forma que obedeça a relação $W_{\text {Caso } 2}^{\text {Má }}>W^{(2)}>W_{\text {Caso } 1}^{\text {Máx }}$ não violaria a Segunda Lei da Termodinâmica e produziria os mesmos resultados inesperados. Esta seção nos permite concluir que:

\section{A forma geral da Segunda Lei da Termodi- nâmica não é suficiente para impedir a exis- tência de um processo cíclico irreversível que seja realizado com eficiência maior do que o processo reversível entre os mesmos estados.}

É somente quando analisamos o balanço de energia do processo que conseguimos encontrar a origem desta inconsistência. Na próxima seção mostraremos, formalmente, que a suposição de reversibilidade $\left(\Delta S^{(2)}=0\right)$ leva à violação da Primeira Lei da Termodinâmica.

\subsection{Caso 3: Processo inverso com refrigeradores de Carnot e compressão isotérmica.}

O objetivo desta seção é mostrar a origem da inconsistência apontada na seção anterior, quando assumimos a reversibilidade do processo descrito no Caso 2. Com esta finalidade construímos um processo reversível (denominado Caso 3) que recupera as condições iniciais do Caso 2 por meio da operação de refrigeradores de Carnot e da execução de uma sucessão de contrações isotérmicas infinitesimais (ver Figura 4). Além disso, conectamos este processo ao Caso 2, com o objetivo de construir um processo cíclico (Caso $2+$ Caso 3 )(ver Figura 5 ).

As contrações isotérmicas do Caso 3 devem ocorrer por meio da liberação de calor para um conjunto de reservatórios térmicos infinitos auxiliares, cada um deles à mesma temperatura do reservatório 1 para cada um dos refrigeradores de Carnot infinitesimais. Esta é a única maneira de garantirmos que a contração isotérmica ocorra de forma reversível.

Portanto, não podemos desconsiderar a existência dos reservatórios auxiliares no Caso 3, uma vez que sem eles, não seria possível a execução das contrações isotérmicas sem que houvesse violação da conservação da energia. Por esta razão, a variação da energia interna no sistema que compõe o Caso 3 deve ser igual à variação da energia interna de todos os reservatórios, ou seja,

$$
\Delta U^{(3)}=\Delta U_{1}^{(3)}+\Delta U_{2}^{(3)}+\Delta U_{3}^{(3)},
$$

onde $\Delta U_{1}^{(3)}=Q_{1}^{(3)}, \Delta U_{2}^{(3)}=Q_{2}^{(3)}$ e $\Delta U_{3}^{(3)}$ é a variação de energia interna nos reservatórios auxiliares. As quantidades $Q_{1}^{(3)}$ e $Q_{2}^{(3)}$ correspondem aos calores líquidos transferidos do refrigerador de Carnot ao reservatório 1 e do reservatório 2 aos refrigeradores, respectivamente. É muito importante destacar que a variação da energia interna do reservatório 1 no Caso $3, \Delta U_{1}^{(3)}$, ocorre 


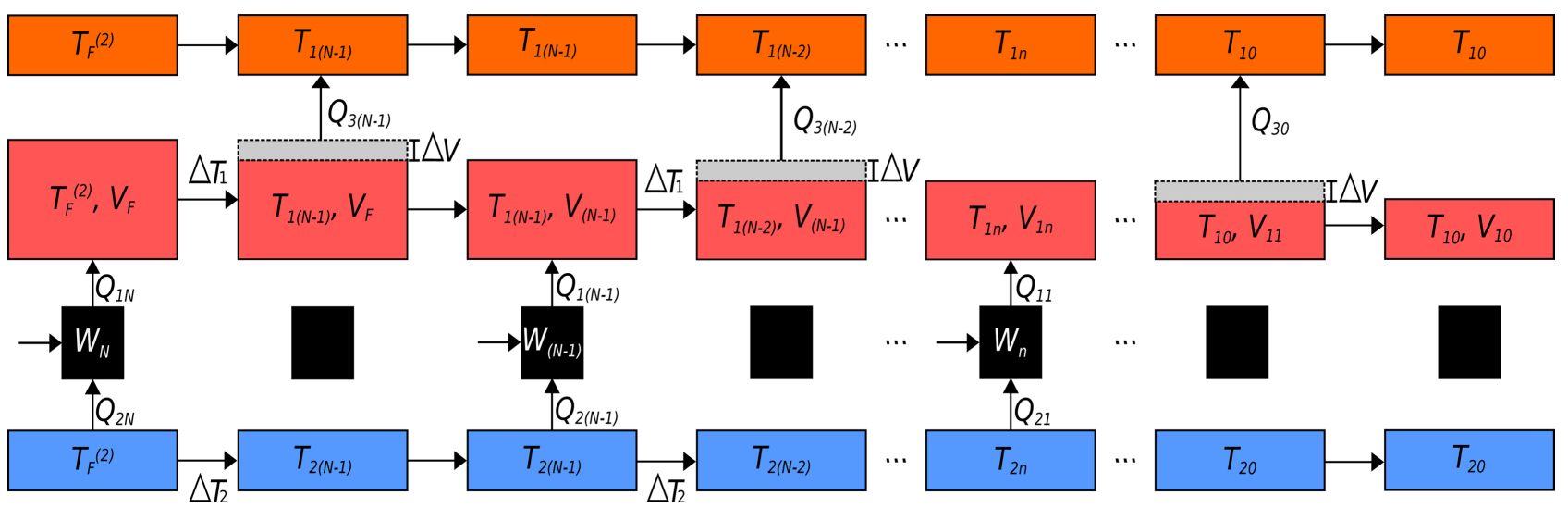

Figura 4: Processo inverso dividido em $N$ etapas discretas, realizadas por refrigeradores de Carnot. Os reservatórios 1,2 e auxiliares foram representados nas cores vermelho, azul e laranja, respectivamente.

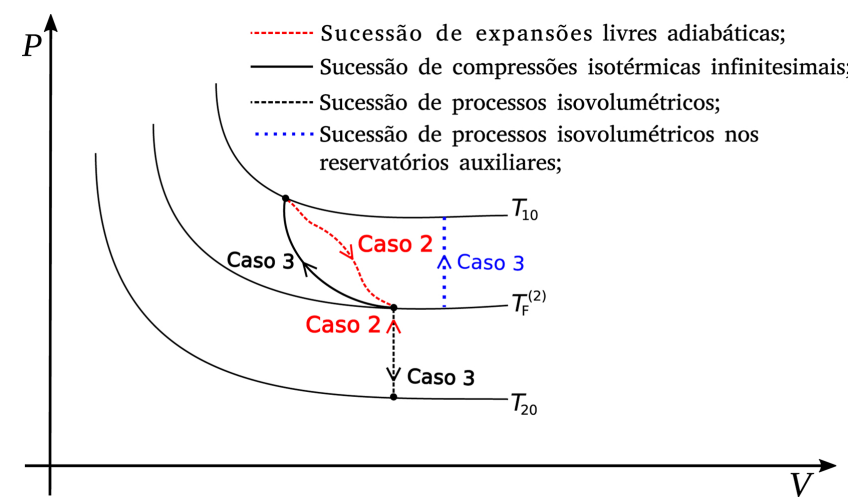

Figura 5: Representação dos processos cíclicos de cada um dos reservatórios. Entre as isotermas $T_{10}$ e $T_{F}^{(2)}$ observa-se o processo cíclico realizado pelo reservatório 1 . Entre as isotermas $T_{20} \mathrm{e}$ $T_{F}^{(2)}$ representamos o processo cíclico associado ao reservatório 2 . A linha pontilhada azul, corresponde à variação de temperatura isovolumétrica nos reservatórios auxiliares do Caso 3.

exclusivamente por meio da atuação do refrigerador de Carnot, uma vez que a contração isotérmica não altera a temperatura do reservatório. Por outro lado, é fácil verificar [4] que para a contração isotérmica de um gás ideal a variação da entropia é não nula. De fato, em cada fase de contração do Caso 3, a variação da entropia é dada por

$$
d S_{1_{n}}^{(3)}=\frac{N_{0} R}{V_{n}} d V \Longrightarrow \Delta S_{1_{n}}^{(3)}=N_{0} R \ln \left(\frac{V_{n+1}}{V_{n}}\right)<0,
$$

pois $V_{n+1}<V_{n}$. No entanto, como por hipótese a contração é feita de forma reversível, a variação da entropia no processo $\left(\Delta S^{(3)}\right)$ tem que ser nula. Isto implica o reservatório auxiliar ter que aumentar sua entropia $\left(\Delta S_{3_{n}}^{(3)}\right)$ de tal forma que a entropia da n-ésima fase do Caso 3 $\left(\Delta S_{n}^{(3)}\right)$ seja

$$
\Delta S_{n}^{(3)}=\Delta S_{1_{n}}^{(3)}+\Delta S_{3_{n}}^{(3)}=0
$$

logo, em cada fase, devemos ter

$$
\Delta S_{3_{n}}^{(3)}=-\Delta S_{1_{n}}^{(3)}>0 .
$$

A condição (35), como consequência da hipótese de reversibilidade da contração isotérmica, impõe um fluxo líquido de calor do reservatório 1 para o reservatório auxiliar, ou seja, devemos ter

$$
\Delta U_{3}^{(3)}=\sum_{n=0}^{N} Q_{3_{n}}^{(3)}=\sum_{n=0}^{N} T_{1_{n}} \Delta S_{3_{n}}^{(3)} \neq 0 .
$$

Por sua vez, no Caso 2, a variação de energia do sistema completo é dada por

$$
\Delta U^{(2)}=Q_{1}^{(2)}+Q_{2}^{(2)}
$$

uma vez que a expansão livre do reservatório 1 é feita sem a realização de trabalho. Neste caso, os reservatórios auxiliares permanecem inalterados, dado que a expansão livre não realiza trabalho e não altera a temperatura do reservatório 1 .

Como as temperaturas finais de cada reservatório do Caso 3 são idênticas às temperaturas iniciais do Caso 2 (e vice-versa) tem-se que

$$
Q_{1}^{(3)}=N_{0} C_{V}\left(T_{10}-T_{F}^{(2)}\right)=-Q_{1}^{(2)}
$$

$\mathrm{e}$

$$
Q_{2}^{(3)}=N_{0} C_{V}\left(T_{20}-T_{F}^{(2)}\right)=-Q_{2}^{(2)} .
$$

Estamos, neste ponto, aptos a calcular a variação da energia interna do sistema quando conectamos os Casos 2 e 3, pois o nosso objetivo é retomar os estados iniciais do Caso 2 por meio do Caso 3. Com o resultado anterior em mente, a partir das equações (32), (36), 37), (38) e (39), a variação total da energia interna $\left(\Delta U_{T}\right)$ do processo completo é dado por

$$
\Delta U_{T}=\Delta U^{(2)}+\Delta U^{(3)}=\Delta U_{3}^{(3)} \neq 0 .
$$

A expressão 40 indica que o processo completo (Caso $2+$ Caso 3) não é cíclico. Isto decorre do fato de existir 
fluxo de calor para o reservatório auxiliar, necessário para que as contrações isotérmicas sejam reversíveis. Por outro lado, se supormos um processo cíclico onde a energia é conservada, ou seja, se assumirmos que

$$
\Delta U_{T}=\Delta U_{3}^{(3)}=0,
$$

então, em decorrência da equação (36), deveríamos obter,

$$
\Delta S_{3_{n}}^{(3)}=0
$$

Aqui nota-se uma inconsistência entre as equações (35) e (42). Além disso, através de (36) vemos que 42 implica

$$
Q_{3_{n}}^{(3)}=0,
$$

ou seja, não haveria fluxo de calor nos processos de contração isotérmica para os reservatórios auxiliares. Nesta situação, como no limite quase estático as máquinas de Carnot operam reversivelmente, através da equação (34) notamos que a variação total de entropia no Caso 3 corresponderia unicamente à entropia produzida durante as contrações isotérmicas,

$$
\Delta S^{(3)}=\sum_{n} \Delta S_{1_{n}}^{(3)}<0
$$

consequência do fato de $\Delta S_{1_{n}}^{(3)}<0$ em cada fase. Desta forma o resultado líquido do processo do Caso 3 seria a violação da Segunda Lei da Termodinâmica. Obviamente isto impede a construção de um processo cíclico que retome os estados iniciais do Caso 2.

As equações (40) e 41) nos deixam apenas duas opções:

(1) Se assumirmos a reversibilidade dos Casos 2 e 3 $\left(\Delta S_{T}=\Delta S^{(2)}+\Delta S^{(3)}=0\right)$ encontramos que o processo não pode ser cíclico, pois violaria a conservação da energia $\left(\Delta U_{T} \neq 0\right)$;

(2) Se assumirmos a conservação da energia $\left(\Delta U_{T}=0\right)$ para o processo cíclico, a Segunda Lei da Termodinâmica seria violada no Caso $3\left(\Delta S^{(3)}<0 \Longrightarrow\right.$ $\left.\Delta S_{T}<0\right)$ e, portanto, não conseguiríamos construir um processo cíclico.

As duas afirmações acima envolvem inconsistências em relação às leis da Termodinâmica.

Estas inconsistências aparecem porque assumimos que um processo envolvendo a expansão livre adiabática pode ser considerado reversível no limite quase estático. Portanto, como esta hipótese gera contradições, ela deve ser falsa.

Desta maneira, mostramos formalmente que a expansão livre adiabática é um processo fundamentalmente irreversível, independentemente da velocidade da expansão. Neste problema, verificamos que a reversibilidade e a conservação da energia são mutuamente excludentes no caso de processos envolvendo máquinas térmicas entre os mesmos reservatórios e sujeitas à expansão livre adiabática infinitesimal. Isto significa dizer:

\begin{abstract}
É impossível que um processo envolvendo a expansão livre adiabática seja reversível, pois, sob esta hipótese, mostramos que existe um processo em que a Primeira e a Segunda leis da Termodinâmica não podem ser satisfeitas simultaneamente.
\end{abstract}

Mas estas não são as únicas inconsistências que emergem neste problema. De fato, no Apêndice $\mathrm{C}$ mostramos que a quantidade de energia degradada pelo processo irreversível com expansão livre é numericamente igual à variação de energia livre do Caso $2, \Delta F_{1}^{(2)}$. É importante notar que o calor transferido para os reservatórios auxiliares nas fases de contração isotérmica sob a hipótese de reversibilidade é exatamente a energia degradada no caso irreversível, ou seja,

$$
\Delta F_{1}^{(2)}=-\Delta U_{3}^{(3)} \neq 0 .
$$

Isto significa que, independentemente da hipótese de reversibilidade, esta energia não pode ser utilizada para realização de trabalho pelo motor.

A Figura 6, justificada no Apêndice D, evidencia que as inconsistências desta seção não estão associadas exclusivamente à hipótese de reversibilidade da expansão livre (ela é apenas um caso particular), mas sim à hipótese de que é possível realizar um trabalho maior do que o trabalho máximo realizado reversivelmente no Caso 1. Se assumirmos que

$$
W_{\text {Caso 2 }}^{\text {Máx }}>W^{(2)}>W_{\text {Caso 1 }}^{\text {Máx }},
$$

então, analogamente ao que foi feito no caso reversível, poderíamos construir um processo cíclico para o qual as leis da Termodinâmica não poderiam ser satisfeitas simultaneamente.

É importante reforçar que a violação da conservação da energia no processo cíclico ocorre por causa da necessidade do fluxo de calor para os reservatórios auxiliares nos casos em que $W^{(2)}>W_{\text {Caso } 1}^{\text {Máx }}$. Nos resta, portanto, avaliar o caso em que

$$
W^{(2)}=W_{\text {Caso } 1}^{\text {Máx }} .
$$

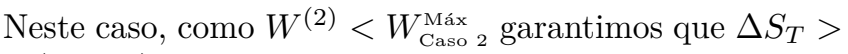
0 (eq. 27). Por outro lado, ao assumirmos a igualdade

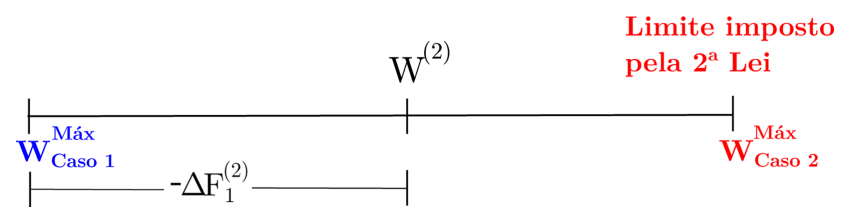

Figura 6: Sob a hipótese de que a Segunda Lei determina o limite de trabalho para o motor no Caso 2, eq. (43), verifica-se que a diferença entre os trabalhos dos Casos 1 e 2 tem íntima relação com variação da energia livre do sistema $\left(\Delta F_{1}^{(2)}\right)$, calculada no Apêndice D. 
acima, de acordo com a Figura 6] e com a equação D1 demonstrada no Apêndice $\mathrm{D}$, verifica-se que $\Delta F_{1}^{(2)}=0$ e, de acordo com o Apêndice C, não há fluxo de calor para os reservatórios auxiliares. Desta forma o processo completo passa a ser cíclico e $\Delta U_{T}=0$. Sob estas condições, nenhuma das leis da Termodinâmica em suas formulações gerais foi violada. A análise deste caso nos permite dizer que um motor utilizando-se de uma expansão livre adiabática poderia operar entre os mesmos estados com a mesma eficiência de uma máquina reversível. Em outras palavras, o resultado anterior nos leva a surpreendente afirmação:

\section{É possível, segundo as formas gerais da $1^{\underline{a}}$ e $2^{\underline{a}}$ leis, que um processo cíclico irre- versivel possua a mesma eficiência de um processo reversível operando entre os mesmos estados.}

Isto soa absurdo, mas surpreendentemente não há qualquer violação das leis da termodinâmica em suas formas gerais. Portanto, o problema requer um cuidado extra em sua análise, pois estamos lidando com os efeitos da (segundo as palavras de Callen [1, problema 4-2.3) "infame"expansão livre adiabática quase estática. É neste ponto que devemos analisar com mais cuidado a expressão

$$
W^{(2)}=N_{0} C_{V}\left(T_{1}+T_{2}-2 \sqrt{T_{1} T_{2}}\left(\frac{V_{0}}{V_{F}^{\prime}}\right)^{\frac{R}{2 C_{V}}}\right),
$$

onde, o volume final do reservatório $1\left(V_{F}^{\prime}\right)$ é tal que $V_{F}>V_{F}^{\prime}>V_{0}$. Perceba que o resultado acima só é válido no limite termodinâmico, ou seja, quando o número de partículas é suficientemente grande. Reescrevemos a expressão (44) como

$$
W^{(2)}=N_{0} C_{V}\left[T_{1}+T_{2}-2 \sqrt{T_{1} T_{2}}\left(1+\frac{\Delta V}{V_{0}}\right)^{-\frac{1}{3}}\right],
$$

onde consideramos que $V_{F}^{\prime}=V_{0}+\Delta V, \operatorname{com} \Delta V / V_{0} \approx 0$, e o calor específico molar a volume constante para um gás ideal monoatômico $C_{V}=\frac{3}{2} R$. Como $\Delta V$ é infinitesimal, aplicando a expansão binomial, temos

$$
W^{(2)}=N_{0} C_{V}\left(T_{1}+T_{2}-2 \sqrt{T_{1} T_{2}}\left(1-\frac{1}{3} \frac{\Delta V}{V_{0}}\right)\right) .
$$

Logo, a equação 45, pode ser escrita como

$$
W^{(2)}=W_{\text {Caso } 1}^{\text {Máx }}+\frac{2}{3} \frac{\Delta V}{V_{0}} C_{V} N_{0} \sqrt{T_{1} T_{2}} .
$$

Assumindo que $T_{1} \neq T_{2} \neq 0$ e $N_{0} \neq 0$, verificamos que a única forma de obtermos $W^{(2)}=W_{\text {Caso } 1}^{\text {Máx }}$ é quando $\Delta V=0$, logo a equação 46 mostra a impossibilidade de se realizar um trabalho $W^{(2)}=W_{\text {Caso } 1}^{\text {Máx }}$ caso ocorra a expansão livre. Como a discussão anterior impede que
$W^{(2)}>W_{\text {Caso 1 }}^{\text {Máx }}$, podemos concluir a partir destes resultados que

$$
W^{(2)}<W_{\text {Caso 1 }}^{\text {Máx }}
$$

sempre que houver a expansão livre adiabática. Desta forma, a expressão 47) recupera a noção intuitiva de que a irreversibilidade associada à expansão livre contribui para a diminuição da eficiência do processo. Mas é importante destacar que a $2^{\mathrm{a}}$ lei da termodinâmica, na sua forma geral, é incapaz de invalidar os casos em que

$$
W_{\text {Caso } 2}^{\text {Máx }}>W^{(2)} \geq W_{\text {Caso 1 } 1}^{\text {Máx }} .
$$

Quando $W^{(2)}>W_{\text {Caso } 1}^{\text {Máx }}$ ocorre a violação da $1^{\text {a }}$ lei, ou seja, da conservação da energia. Quando $W^{(2)}=W_{\text {Caso } 1}^{\text {Máx }}$ nenhuma das leis (nas suas formas gerais) é violada, mas a análise explícita do trabalho realizado no processo irreversível nos mostra a impossibilidade de que o processo irreversível não contribua para a diminuição do trabalho realizado pelo sistema. De fato, é fácil verificar que sempre que houver a expansão livre teremos

$$
\Delta U=W^{(2)}-W_{\text {Caso } 1}^{\text {Máx }}=\frac{2}{3} \frac{\Delta V}{V_{0}} C_{V} N_{0} \sqrt{T_{1} T_{2}} \neq 0,
$$

no processo cíclico (Caso 2 + Caso 3), ou seja, haverá violação da Primeira Lei da Termodinâmica.

No entanto, podemos verificar que existem outras três possibilidades de tornar o trabalho com a expansão livre igual ao trabalho reversível sem violarmos a Primeira Lei. Isto é possível, por exemplo, se um dos reservatórios tiver temperatura nula. Se supormos $T_{1}=0$, então não haverá extração de calor e consequentemente a eficiência do processo será nula. Este é um caso trivial. Por outro lado, se supormos $T_{2}=0$, a máquina de Carnot operaria com eficiência dada por

$$
\eta=1-\frac{T_{2}}{T_{1}}=\frac{\left|W_{R}\right|}{\left|Q_{1}\right|}=1
$$

ou seja, violaria a Segunda Lei na forma de Kelvin-Planck. De fato, ao analisarmos a equação 12 , verificamos que, no caso de $T_{2}=0$ não haveria variação de temperatura no reservatório 2 em qualquer uma das etapas infinitesimais, logo o motor converteria toda a energia extraída do reservatório 1 em trabalho.

Uma terceira forma seria tomar $N_{0}=0$. Neste caso, obviamente não teríamos de onde extrair energia. No entanto, se tomarmos $N_{0}$ muito pequeno (por exemplo se tomarmos $10^{6}$ moléculas, teríamos $N_{0} \approx 10^{-17}$ moles), a contribuição associada a expansão livre irreversível seria insignificante, logo

$$
\lim _{N_{0} \rightarrow 0} W^{(2)}=W_{\text {Caso } 1}^{\text {Máx }} .
$$

Embora este resultado pareça uma violação das leis da termodinâmica, o regime de $N_{0}$ baixo está fora do limite usual no qual a termodinâmica é estabelecida, ou seja, 
as suas leis não podem ser enunciadas sem cuidado. Este resultado nos mostra, portanto, que é importante entendermos o comportamento de sistemas com poucos graus de liberdade, dado que trabalhos recentes [15] nos mostram que sistemas clássicos caóticos com poucos graus de liberdade, mesmo que muito longe do limite termodinâmico, podem exibir propriedades termodinâmicas. Isto deixa a mensagem de que o número de partículas que compõem o sistema não é um parâmetro seguro para determinar os limites de validade das leis da termodinâmica.

\section{Conclusões}

Nos livros básicos de termodinâmica é muito comum a discussão de um problema constituído de uma máquina acoplada a dois reservatórios térmicos finitos a temperaturas diferentes. Neste problema, resolvido no Apêndice $A$, verifica-se que a quantidade de trabalho que pode ser produzido por qualquer motor a partir da energia dos reservatórios é limitada por

$$
W \leqslant N_{0} C_{V}\left(T_{1}+T_{2}-2 \sqrt{T_{1} T_{2}}\right) .
$$

O primeiro resultado deste trabalho está associado à verificação do limite superior da equação 49. Isto foi feito no Caso 1, onde tomamos o limite quase estático do processo ao discretizá-lo em $N$ etapas com variações infinitesimais de calor nos reservatórios, por meio da operação de máquinas de Carnot. Esta é uma maneira didática de se mostrar que a igualdade em (49) é alcançada devido às máquinas de Carnot que, neste caso, somente operam de forma reversível no limite infinitesimal (quase estático). Portanto, no Caso 1, a reversibilidade é uma consequência da natureza quase estática do processo.

O próximo passo foi investigar as consequências de se assumir que um processo fundamentalmente irreversível (expansão livre adiabática) pode ser considerado reversível no limite quase estático. Com este intuito, no Caso 2, ainda com o processo discretizado, permitimos que um dos reservatórios sofresse expansões livres adiabáticas infinitesimais entre cada uma das $N$ etapas. Como resultado, obtivemos um limite diferente da equação (49), dado pela expressão

$$
W^{(2)} \leqslant N_{0} C_{V}\left(T_{1}+T_{2}-2\left(T_{1} T_{2}\right)^{\frac{1}{2}}\left(\frac{V_{0}}{V_{F}}\right)^{\frac{R}{2 C_{V}}}\right)
$$

Comparando as expressões 49 e 50 , ao assumirmos a reversibilidade em ambos os casos, concluímos que

$$
W_{\text {Caso } 2}^{\text {Máx }}>W_{\text {Caso } 1}^{\text {Máx }}
$$

e que

$$
\eta_{\text {Caso 2 }}^{\text {Máx }}>\eta_{\text {Caso 1 }}^{\text {Máx }} \text {. }
$$

Estes resultados, absolutamente contra-intuitivos, nascem por meio da hipótese de reversibilidade do processo no qual ocorrem as expansões livres. Cabe aqui um comentário muito importante. Como a Segunda Lei em sua forma geral deve ser aplicada a sistemas fechados, a hipótese de reversibilidade foi aplicada à variação da entropia total do sistema, ou seja, como existe uma fase de troca de energia dos reservatórios com o motor, não podemos considerar os reservatórios como um sistema isolado e, portanto, não podemos analisar a variação da entropia da expansão livre adiabática separadamente. Desta forma, mesmo sabendo que no caso isolado a expansão livre adiabática é fundamentalmente irreversível, pois a variação da entropia depende somente dos estados finais e iniciais (e não da natureza quase estática), os resultados emergentes da hipótese de que o processo completo, incluindo a expansão livre adiabática infinitesimal, é reversível, atestam que seríamos capazes de aumentar sua eficiência.

A estranheza dos resultados do Caso 2, vinculados inicialmente à hipótese de reversibilidade do processo quase estático, sugerem que é possível construir um terceiro processo que, conectado ao Caso 2, viole alguma das Leis da Termodinâmica. Com isto em mente, construímos um processo inverso, denominado Caso 3, realizado de forma reversível que, partindo da configuração final do Caso 2, retornasse ao seu estado inicial (do Caso 2). Para isto realizamos contrações isotérmicas infinitesimais no reservatório 1 em cada etapa do processo discretizado e operamos refrigeradores de Carnot entre as mesmas. Diferentemente do que ocorre no Caso 2, as contrações isotérmicas reversíveis somente podem ocorrer caso haja troca de calor com um reservatório auxiliar. Ao conectarmos os Casos 2 e 3 (ver Figura 5 ) e, assumindo a reversibilidade do processo completo, obtemos que (equação (40) )

$$
\Delta U_{T}=\Delta U_{3}^{(3)} \neq 0
$$

sempre. Isto significa que é impossível retomar os estados iniciais do Caso 2 sem que haja variação da energia interna no reservatório auxiliar. Este resultado mostra que é impossível se construir um processo cíclico, caso assumamos a reversibilidade. Mas isto é um absurdo, pois a reversibilidade implica a possibilidade de se alcançar o estado inicial por meio da reversão do processo original, ou seja, implicaria a possibilidade de execução de um processo cíclico. Neste caso, se assumirmos que o sistema completo recupera o estado inicial do Caso 2 por meio da reversão do processo original com $\Delta S_{T}=0$, a energia do sistema completo não poderia ser conservada.

Em contrapartida, se assumirmos que o sistema completo recupera o estado inicial do Caso 2 por meio da reversão do processo original conservando a energia, ou seja, com $\Delta U_{T}=0$, mostramos que

$$
\Delta S^{(3)}<0,
$$

uma violação na Segunda Lei, pois o sistema é fechado. Consequentemente, não podemos assumir a reversibilidade da conexão dos Casos 2 e 3 sem violar alguma das Leis da Termodinâmica. Portanto, 
É impossivel que o processo do Caso 2, que inclui a expansão livre adiabática, seja reversível, pois, sob esta hipótese, mostramos que existe um processo em que a Primeira e Segunda leis da Termodinâmica não podem ser satisfeitas simultaneamente.

A conclusão acima nos permite afirmar que:

(i) A natureza quase estática de um processo termodinâmico não pode ser considerada como uma condição suficiente para a reversibilidade do mesmo.

A necessidade de utilização da Primeira e Segunda leis da Termodinâmica em conjunto para mostrar a impossibilidade de realização de um processo que seria absurdo, reforça algo muito importante e presente nos cursos mais avançados de Termodinâmica: a entropia e a energia interna são grandezas canonicamente conjugadas por meio de uma Transformação de Legendre [1]. Tanto a energia interna como a entropia contém toda a informação sobre o sistema e são capazes de determinar as regras segundo as quais o sistema evolui. Este trabalho, portanto, evidência indiretamente este aspecto e reforça que a Primeira e Segunda leis da Termodinâmica devem ser analisadas em conjunto quando buscamos avaliar os limites impostos pela reversibilidade.

As inconsistências encontradas no Caso 2 não estão associadas unicamente à hipótese de reversibilidade. Quando avaliamos a possibilidade de realização de um trabalho irreversível $W^{(2)}$ seguindo o protocolo do Caso 2 e maior que o trabalho máximo do Caso 1, concluímos que

(ii) A utilização da forma geral da Segunda Lei da Termodinâmica não é suficiente para evitar que o trabalho realizado em um processo cíclico irreversivel $\left(W^{(2)}\right)$ seja maior do que o realizado em um processo reversivel entre os mesmos estados, ou seja, a análise isolada da Lei $\Delta S \geq 0$ para sistemas fechados não impede que tenhamos

$$
W_{\text {Caso } 2}^{\text {Max }}>W^{(2)}>W_{\text {Caso } 1}^{M a x} .
$$

No entanto, mostramos que, caso a condição acima fosse satisfeita, teríamos uma variação da energia interna dada por $\Delta U_{T}=\Delta U_{3}^{(3)} \neq 0$ no processo cíclico, ou seja, uma violação da Primeira Lei.

A grande dificuldade em se utilizar a forma geral das leis da termodinâmica está associada ao fato de que a Segunda Lei é escrita em termos de uma desigualdade, o que deixa margem para que qualquer quantidade de trabalho decorrente do limite imposto por $\Delta S \geq 0$ seja "permitido". Ainda mostramos que:

(iii) As formas gerais da Primeira e Segunda leis não impedem que o trabalho realizado em um processo cíclico irreversível $\left(W^{(2)}\right)$ seja igual ao de um processo reversivel operando entre os mesmos estados, ou seja,

$$
W^{(2)}=W_{\text {Caso } 1}^{\text {Máx }} \text {. }
$$

No entanto, a análise explícita do trabalho associado ao processo irreversível, equação (46), nos permite concluir que é impossível obtermos a mesma eficiência, caso ocorra a expansão livre. Neste caso, portanto, devemos ter

$$
W^{(2)}<W_{\text {Caso 1 } 1}^{\text {Máx }} \text {. }
$$

Os apêndices C e D evidenciam que a expansão livre diminui a energia livre do sistema, o que reforça a interpretação de que a quantidade de energia útil para a realização de trabalho é menor quando incluímos um processo irreversível. A expressão (46) ainda nos faz vislumbrar as complicações oriundas do baixo número de graus de liberdade, limite no qual a Termodinâmica não está bem estabelecida. Os limites entre reversibilidade e irreversibilidade ficam difusos neste regime e provavelmente o caráter dinâmico do sistema tenha algum papel relevante. Isto nos motiva a fazer as seguintes indagações:

- As leis e propriedades termodinâmicas continuam sendo válidas em escalas microscópicas?

- Como definir reversibilidade ou irreversibilidade nestas escalas?

Este trabalho não tem como intuito mostrar que as Leis da Termodinâmica não são válidas, mas sim reforçar que as suas formas gerais podem conduzir a resultados equivocados nos limites analisados neste trabalho. No entanto, à luz das formulações experimentais (Invariância pelo caminho do Trabalho Adiabático e o Enunciado de Kelvin-Planck) verificamos que é impossível que estas inconsistências persistam. Portanto, esperamos que este trabalho contribua para:

- a verificação de que a realização quase estática de um processo não é uma condição suficiente para a reversibilidade;

- a compreensão de que, muito embora as formas gerais das Leis da Termodinâmica estejam muito bem estabelecidas, a sua utilização inadvertida pode conduzir a resultados absurdos, diferentemente das formulações experimentais.

Enfim, desejamos que este trabalho contribua de alguma forma para o estabelecimento de questões a respeito dos limites de validade da teoria.

\section{Agradecimentos}

Este trabalho foi realizado com o apoio da UFMT e do $\mathrm{CNPq}$ por meio do Programa de Iniciação Científica PIBIC. Os autores agradecem ao Prof. Márcio Fernando Cornélio e ao Prof. Thiago W. de Oliveira, pela leitura 
do manuscrito original. A autora S. S. Dourado, agradece especialmente à amiga Alice Caroline V. de Oliveira pelas valiosas discussões.

\section{Material Suplementar}

O seguinte material suplementar está disponível online:

Apêndice A

Apêndice $\mathrm{B}$

Apêndice $\mathrm{C}$

Apêndice D

\section{Referências}

[1] H.B. Callen, Thermodynamics and an Introduction to Thermostatistics (John Wiley, New York, 1985), $2^{\mathrm{a}}$ ed.

[2] M.W. Zemansky e R.H. Dittman, Heat and Thermodynamics (McGraw-Hill, New York, 1997), $7^{\text {a }}$ ed.

[3] G.J. Van Wylen e R.E. Sonntag, Fundamentos da Termodinâmica Clássica (Edgard Blucher, São Paulo, 1976), $2^{\mathrm{a}}$ ed.

[4] H.M. Nussenzveig, Curso de Física Básica (Edgar Blucher, São Paulo, 2002), v. 2, $4^{\mathrm{a}}$ ed.

[5] R.P. Feynman, R.B. Leighton e M. Sands, Feynman Lições de Física (Bookman, Porto Alegre, 2008), v. 1, 1aㅗ ed.

[6] C. Van den Broeck, Phys. Rev. Lett. 95, 190602 (2005).

[7] B. Andresen, P. Salamon e R.S. Berry, Phys. Today 37, $62(1984)$.

[8] A.E. Allahverdyan, K.V. Hovhannisyan, A.V. Melkikh e S.G. Gevorkian, Phys. Rev. Lett. 111, 050601 (2013).

[9] M. Esposito, R. Kawai, K. Lindenberg e C. Van den Broeck, Phys. Rev. Lett. 105, 150603 (2010).

[10] F. Curzon e B. Ahlborn, Am. J. Phys. 43, 22 (1975).

[11] J.F. Stilck e R.M. Brum, Rev. Bras. Ens. Fís. 35, 1 (2013).

[12] R. Landauer, IBM J. Res. Dev. 5, 183 (1961).

[13] C.H. Bennett, Stud. Hist. Philos. Sci. B: Stud. Hist. Philos. Mod. Phys. 34, 501 (2003).

[14] C.H. Bennett, Int. J. Theor. Phys. 21, 905 (1982).

[15] M.A. Marchiori e M.A.M. de Aguiar, Phys. Rev, E. 83, 061112 (2011). 